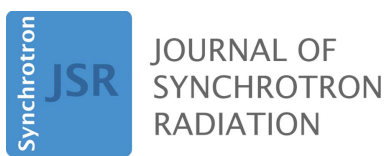

ISSN 1600-5775

Received 29 June 2018

Accepted 9 October 2018

Edited by A. F. Craievich, University of São Paulo, Brazil

Keywords: multi-element X-ray movie imaging; CMOS camera.

Supporting information: this article has supporting information at journals.iucr.org/s

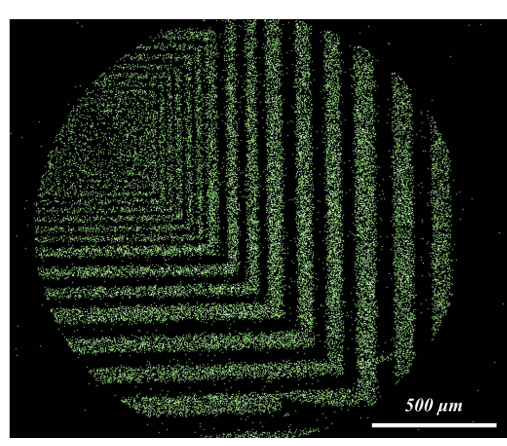

OPEN $\odot$ ACCESS

\section{Multi-element X-ray movie imaging with a visible-light CMOS camera}

\author{
Wenyang Zhao ${ }^{a, b}$ and Kenji Sakurai ${ }^{b, a *}$ \\ aUniversity of Tsukuba, 1-1-1 Tennodai, Tsukuba, Ibaraki 305-0006, Japan, and ${ }^{\mathbf{b}}$ National Institute for Materials \\ Science, 1-2-1 Sengen, Tsukuba, Ibaraki 305-0047, Japan. *Correspondence e-mail: sakurai@yuhgiri.nims.go.jp
}

For many years, X-ray movies have been considered a promising tool for exploring and providing insights into chemical reactions. A simultaneous multielement X-ray movie can further clarify the behavior difference of various elements and help investigate their interactions. The present short communication illustrates how to conduct multi-element X-ray movie imaging in a synchrotron facility solely by placing a micro-pinhole in front of a visible-light complementary metal-oxide semiconductor (CMOS) camera. It has been found that the CMOS camera can resolve X-ray fluorescence spectra when it is specially operated. In this work, a spatial resolution of $\sim 15 \mu \mathrm{m}$ was achieved. In the X-ray movie, a movie frame acquisition time of $2 \mathrm{~min}$ and a spatial resolution of $\sim 50 \mu \mathrm{m}$ were simultaneously achieved. It is clear that the CMOS camera can be a cost-efficient option for many researchers who wish to establish their own setup for visualizing chemical diffusion in various reactions.

\section{Introduction}

Movies are useful as a record of the overall progress of chemical reactions. Generally, a movie captured in visible light cannot reveal the elemental composition of a reaction. By contrast, X-ray fluorescence (XRF) can aid the identification of elements with quantitative information such as average concentration, and XRF imaging can reveal their spatial distribution. Therefore, a movie comprising a continuous series of XRF images can be a powerful tool for exploring multi-element reactions such as ion exchange, composition segregation, selective precipitation and formation of chemicalgradient materials (Zhao \& Sakurai, 2017a).

Full-field XRF imaging is an efficient way to collect XRF images. The technique requires an X-ray area detector that also possesses spectroscopic capability. Thus far, several options exist including a pn charge-coupled device (CCD) (Strüder et al., 2001; Scharf et al., 2011) and single-photoncounting CCD (Romano et al., 2014, 2016; Zhao \& Sakurai, $2017 b$ ). The present short communication indicates the costefficient alternative of a visible-light complementary metaloxide semiconductor (CMOS) camera used for direct detection of X-rays (Janesick, 2001; Holst \& Lomheim, 2007). In previous work, it was found that the camera could resolve XRF spectra using laboratory X-ray sources (Zhao \& Sakurai, 2017c). Here, the camera operation is performed in a synchrotron facility and its multi-element movie imaging capability with potential for high spatial resolution is illustrated.

\section{Experimental}

The visible-light CMOS camera (PCO.edge 5.5, PCO AG) used in the present work has $2560 \times 2160$ pixels with a pixel 
pitch of $6.5 \mu \mathrm{m} \times 6.5 \mu \mathrm{m}$. The sensor was electrically cooled down to $5^{\circ} \mathrm{C}$. For full-field $\mathrm{X}$-ray imaging, the optical window of the camera was replaced with a $25 \mu \mathrm{m}$-thick beryllium $\mathrm{X}$-ray window. A pinhole was drilled onto $50 \mu \mathrm{m}$-thick tungsten foil, which was then installed in front of the X-ray window. To measure the X-ray photon energies, the camera was operated in single-photon-counting mode, and all camera images were processed using an integrated-filtering method (Zhao \& Sakurai, 2017c) for charge-sharing correction.

Experiments were conducted in the BL-14B Photon Factory of KEK, Japan. The primary X-ray beam passed through an $\mathrm{Si}(111)$ double-crystal monochromator and was collimated to a width of $1 \mathrm{~mm}$ and height of $10 \mathrm{~mm}$. The energy used in the present work was $13.5 \mathrm{keV}$. In the imaging experiments, samples were positioned vertically with a glancing angle of approximately $5^{\circ}$ between the primary X-ray beam and the sample surface (Fig. S1 of the supporting information).

Two additional modifications were necessary for applying the camera to synchrotron experiments. First, the top part of the camera housing was changed from aluminium to $2 \mathrm{~mm}$ thick brass for shielding against high-energy X-ray scattering. Second, the double-crystal monochromator was detuned to eliminate higher-order harmonics that contribute strongly to noise collected by the camera sensor.

\section{Results and discussion}

The spatial resolution of the full-field XRF imaging system was tested using a pinhole diameter of $10 \mu \mathrm{m}$. The distances from the pinhole to the target and camera sensor were $1.65 \mathrm{~mm}$ and $14 \mathrm{~mm}$, respectively. The resolution target was a chromium pattern coated on silica glass [Fig. 1(a)]. The total measurement time was $6 \mathrm{~h}$. Subsequent full-field XRF analysis [Fig. 1(b)] shows the spectral peaks of chromium from the target. XRF contamination of copper and zinc, which originated from the brass housing of the camera, was also observed. The XRF image of chromium [Fig. 1(c)] indicates a spatial resolution of $15 \mu \mathrm{m}$ or even better for the system.

To demonstrate multi-element X-ray movie imaging, the growth process of zinc dendrites during electro-deposition was recorded. The reaction occurred in a thin container with dimensions of $30 \mathrm{~mm}(\mathrm{~W}) \times 20 \mathrm{~mm}(\mathrm{~L}) \times 1 \mathrm{~mm}(\mathrm{D})$. The container was filled with $0.1 M \mathrm{ZnCl}_{2}$ solution, and two copper electrodes were immersed (Fig. S1). The distance from the upper tip of the cathode to the lower horizontal anode wire was $15 \mathrm{~mm}$. The electric potential between them was $3 \mathrm{~V}$. The front surface of the container was made of a $50 \mu \mathrm{m}$-thick polyester film to allow the X-rays to pass through. In this experiment, a $50 \mu \mathrm{m}$ pinhole was used to collect a stronger $\mathrm{XRF}$ signal although this reduces the spatial resolution. The distances from the pinhole to the reaction cell and camera sensor were $8 \mathrm{~mm}$ and $14 \mathrm{~mm}$, respectively. After connecting the circuit to initiate the reaction, the experiment continued for $1 \mathrm{~h}$, as did the movie recording. In the full-field XRF spectra [Fig. 2(a)], most events in the $\mathrm{Zn} K \alpha$ peak came from the reaction cell (not the brass shielding), and therefore the spectral imaging of $\mathrm{Zn} K \alpha$ showed the zinc distribution in the

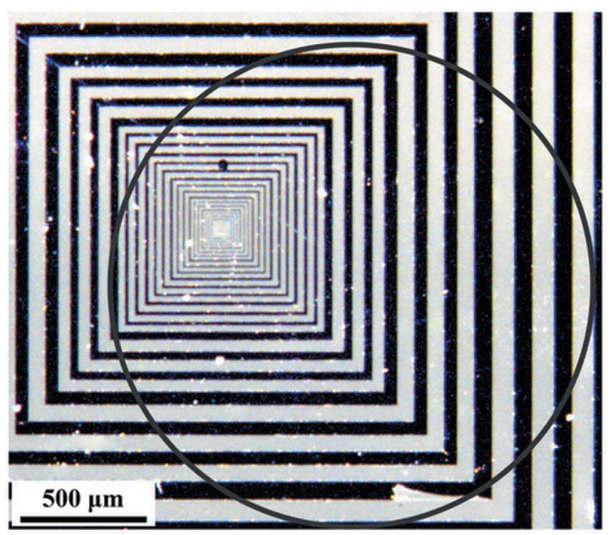

(a)

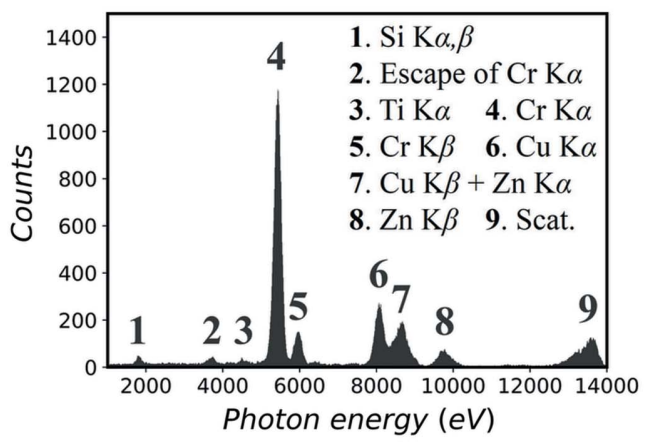

(b)

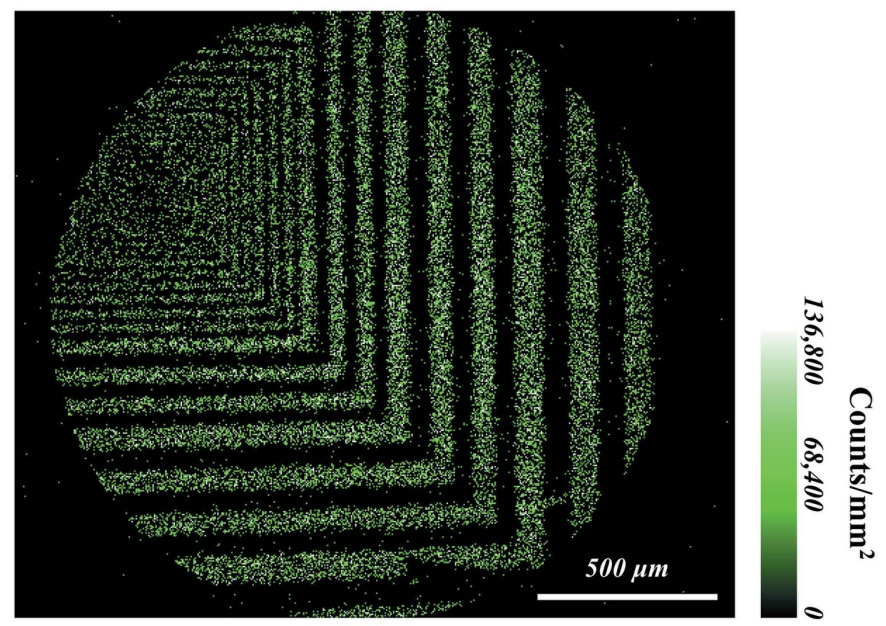

(c)

Figure 1

Full-field XRF imaging of a commercial-resolution target sample. (a) An optical photograph of the resolution target. The black rectangular patterns are chromium coatings. The XRF imaging area is enclosed by the circle and the scale bar is $500 \mu \mathrm{m}$. (b) Full-field XRF spectra accumulated for 6 h. (c) An XRF image of $\mathrm{Cr} K \alpha$ and $K \beta$.

reaction. In this way, images of zinc were generated every $2 \mathrm{~min}$, and a continuous movie was obtained [Fig. 2(b)]. These XRF movie frames confirm the visual observation that zinc dendrites first appeared on the upper tip of the cathode and then gradually grew downward; they also show what visible light cannot: the zinc dispersed in the solution and was gradually exhausted. 


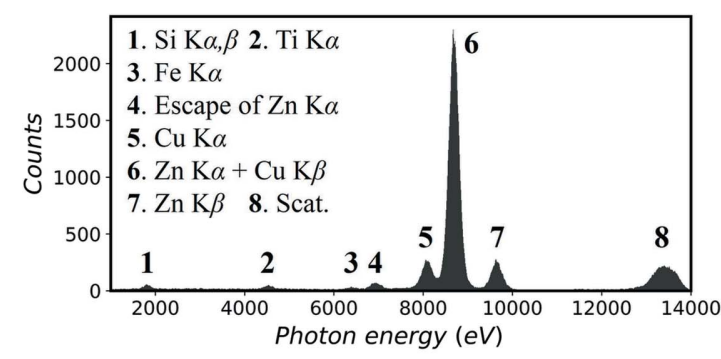

(a)
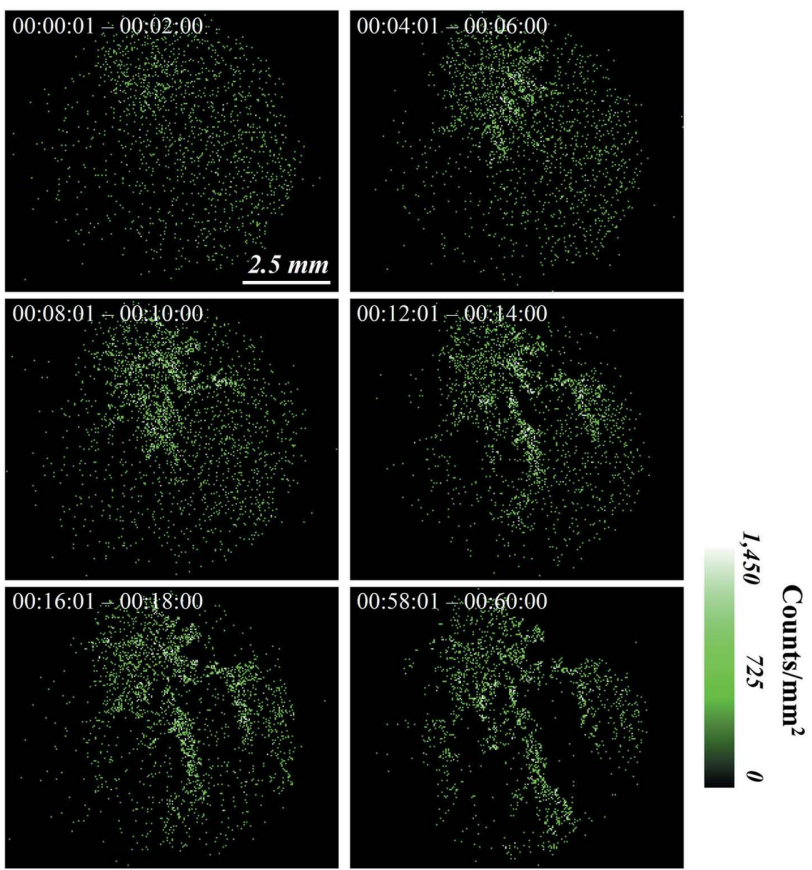

(b)

Figure 2

Application to observation of growing zinc electrodeposits. (a) Full-field XRF spectra. (b) Some key frames of the movie. The acquisition time for one frame is $2 \mathrm{~min}$.

In the spectra shown in Figs. 1(b) and 2(a), the small peak of Si $K \alpha, \beta$ originated from the Si-based camera sensor. The small peaks of Ti $K \alpha$ and Fe $K \alpha$ are assigned to trace environmental contaminants. Furthermore, in this work the XRF spectra have a slight spectral contamination of zinc and copper from the brass camera housing. In future, this contamination can be easily eliminated by optimizing the housing shape or coating other high- $Z$ 'XRF-silent' elements. Meanwhile, the present work shows that the spatial resolution of full-field XRF imaging can reach $15 \mu \mathrm{m}$ by using a pinhole of a sufficiently small size. When employing this simple approach, the XRF intensity collected by the pinhole becomes weaker as the pinhole becomes smaller. Consequently, a larger pinhole was adopted in the movie experiment as a compromise. To reduce this problem, a higher-flux synchrotron X-ray beam may be used to compensate the intensity loss, or the pinhole may be replaced by other X-ray imaging optics such as a collimator plate (Sakurai, 1999; Sakurai \& Eba, 2003; Sakurai \& Mizusawa, 2004; Mizusawa \& Sakurai, 2004; Eba et al., 2016). Other approaches explore the use of polycapillaries (Scharf et al.,
2011), modified uniformly redundant array masks (Haboub et $a l ., 2014)$ and potentially other devices in this context.

The silicon-based image sensor of the CMOS camera was estimated to be thinner than $10 \mu \mathrm{m}$ by measuring and comparing its absorption efficiency for X-rays of different energies. Therefore, the visible-light CMOS camera has a lower detection efficiency for high-energy X-rays compared with many professional X-ray area detectors (Strüder et al., 2001; Henrich et al., 2009; Dinapoli et al., 2011; Blaj et al., 2016). However, its low cost and contemporary availability provides an excellent opportunity for many researchers to establish their own setups. Moreover, considering its unique advantages of a large pixel count and small pixel size, it is clear that the CMOS camera has potential as the XRF detector of choice for visualizing chemical diffusion in various reactions.

\section{Acknowledgements}

The authors would like to thank Professor Keiichi Hirano (KEK, Photon Factory) for his kind assistance during the experiments.

\section{Funding information}

This work was carried out with the approval of the Photon Factory Program Advisory Committee (proposal No. 2018 G030). This work was partly supported by JSPS KEKENHI (grant No. JP18J12732).

\section{References}

Blaj, G., Caragiulo, P., Carini, G., Dragone, A., Haller, G., Hart, P., Hasi, J., Herbst, R., Kenney, C., Markovic, B., Nishimura, K., Pines, J., Segal, J., Tamma, C. \& Tomada, A. (2016). AIP Conf. Proc. 1741, 040012.

Dinapoli, R., Bergamaschi, A., Henrich, B., Horisberger, R., Johnson, I., Mozzanica, A., Schmid, E., Schmitt, B., Schreiber, A., Shi, X. \& Theidel, G. (2011). Nucl. Instrum. Methods Phys. Res. A, 650, 7983.

Eba, H., Ooyama, H. \& Sakurai, K. (2016). J. Anal. At. Spectrom. 31, 1105-1111.

Haboub, A., MacDowell, A. A., Marchesini, S. \& Parkinson, D. Y. (2014). Rev. Sci. Instrum. 85, 063704.

Henrich, B., Bergamaschi, A., Broennimann, C., Dinapoli, R., Eikenberry, E. F., Johnson, I., Kobas, M., Kraft, P., Mozzanica, A. \& Schmitt, B. (2009). Nucl. Instrum. Methods Phys. Res. A, 607, 247-249.

Holst, G. C. \& Lomheim, T. S. (2007). CMOS/CCD Sensors and Camera Systems. Washington: SPIE.

Janesick, J. R. (2001). Scientific Charge-Coupled Devices. Washington: SPIE.

Mizusawa, M. \& Sakurai, K. (2004). J. Synchrotron Rad. 11, 209-213.

Romano, F. P., Caliri, C., Cosentino, L., Gammino, S., Giuntini, L., Mascali, D., Neri, L., Pappalardo, L., Rizzo, F. \& Taccetti, F. (2014). Anal. Chem. 86, 10892-10899.

Romano, F. P., Caliri, C., Cosentino, L., Gammino, S., Mascali, D., Pappalardo, L., Rizzo, F., Scharf, O. \& Santos, H. C. (2016). Anal. Chem. 88, 9873-9880.

Sakurai, K. (1999). At. Spectrosc. 54, 1497-1503.

Sakurai, K. \& Eba, H. (2003). Anal. Chem. 75, 355-359.

Sakurai, K. \& Mizusawa, M. (2004). AIP Conf. Proc. 705, 889-892.

Scharf, O., Ihle, S., Ordavo, I., Arkadiev, V., Bjeoumikhov, A., Bjeoumikhova, S., Buzanich, G., Gubzhokov, R., Günther, A., Hartmann, R., Kühbacher, M., Lang, M., Langhoff, N., Liebel, A., 
Radtke, M., Reinholz, U., Riesemeier, H., Soltau, H., Strüder, L., Thünemann, A. F. \& Wedell, R. (2011). Anal. Chem. 83, 2532-2538. Strüder, L., Briel, U., Dennerl, K., Hartmann, R., Kendziorra, E., Meidinger, N., Pfeffermann, E., Reppin, C., Aschenbach, B., Bornemann, W., Bräuninger, H., Burkert, W., Elender, M., Freyberg, M., Haberl, F., Hartner, G., Heuschmann, F., Hippmann, H., Kastelic, E., Kemmer, S., Kettenring, G., Kink, W., Krause, N., Müller, S., Oppitz, A., Pietsch, W., Popp, M., Predehl, P., Read, A., Stephan, K. H., Stötter, D., Trümper, J., Holl, P., Kemmer, J., Soltau,
H., Stötter, R., Weber, U., Weichert, U., von Zanthier, C., Carathanassis, D., Lutz, G., Richter, R. H., Solc, P., Böttcher, H., Kuster, M., Staubert, R., Abbey, A., Holland, A., Turner, M., Balasini, M., Bignami, G. F., La Palombara, N., Villa, G., Buttler, W., Gianini, F., Lainé, R., Lumb, D. \& Dhez, P. (2001). Astron. Astrophys, 365, L18-L26.

Zhao, W. \& Sakurai, K. (2017a). ACS Omega, 2, 4363-4369.

Zhao, W. \& Sakurai, K. (2017b). Rev. Sci. Instrum. 88, 063703.

Zhao, W. \& Sakurai, K. (2017c). Sci. Rep. 7, 45472. 